\title{
Design and Performance of a TES X-ray Microcalorimeter Array for Energy Dispersive Spectroscopy on Scanning Transmission Electron Microscope
}

\author{
Haruka Muramatsu ${ }^{1}$ - K. Nagayoshi ${ }^{1}$ - T. Hayashi ${ }^{1}$. \\ K. Sakai ${ }^{1}$ - R. Yamamoto ${ }^{1}$. K. Mitsuda ${ }^{1}$. \\ N. Y. Yamasaki ${ }^{1}$ - K. Maehata ${ }^{2}$. T. Hara ${ }^{3}$
}

Received: 14 October 2015 / Accepted: 3 February 2016 / Published online: 18 February 2016 (C) The Author(s) 2016. This article is published with open access at Springerlink.com

\begin{abstract}
We discuss the design and performance of a transition edge sensor (TES) $\mathrm{X}$-ray microcalorimeter array for scanning transmission electron microscope (STEM)energy dispersive X-ray spectroscopy (EDS). The TES X-ray microcalorimeter has better energy resolution compared to conventional silicon drift detector and STEMEDS utilizing a TES detector makes it possible to map the distribution of elements on a specimen in addition to analyze the composition. The requirement for a TES detector is a high counting rate $(>20 \mathrm{kcps})$, wide energy band $(0.5-15 \mathrm{keV})$ and good energy resolution $(<10 \mathrm{eV})$ full width at half maximum. The major improvement of this development is to increase the maximum counting rate. In order to accommodate the high counting rate, we adopted an $8 \times 8$ format, 64-pixel array and common biasing scheme for the readout method. We did all design and fabrication of the device in house. With the device we have fabricated most recently, the pulse decay time is $40 \mu \mathrm{s}$ which is expected to achieve $50 \mathrm{kcps}$. For a single pixel, the measured energy resolution was $7.8 \mathrm{eV}$ at $5.9 \mathrm{keV}$. This device satisfies the requirements of counting rate and energy resolution, although several issues remain where the performance must be confirmed.
\end{abstract}

Keywords TES X-ray microcalorimeter $\cdot$ STEM $\cdot$ EDS

Haruka Muramatsu

muramatu@astro.isas.jaxa.jp

1 Institute of Space and Astronautical Science, Japan Aerospace Exploration Agency (ISAS/JAXA), 3-1-1 Yoshinodai, Chuo, Sagamihara, Kanazawa 252-5210, Japan

2 Department of Applied Quantum Physics and Nuclear Engineering, Kyusyu University, 744 Motooka, Nishi-ku, Fukuoka, Fukuoka 819-0395, Japan

3 National Institute for Materials Science (NIMS), 1-1, Namiki, Tsukuba, Ibaraki 305-0044, Japan 


\section{Introduction}

An EDS performed with electron microscopes now plays an important role for nanoscale compositional analysis in various fields such as material science and biotechnology. In this experiment, an electron beam is directed to a specimen and the elements in the specimen are determined by measuring the energy of their characteristic X-rays. In this way, we are able to detect all most of the elements at the same time. Presently, Si solid-state detectors are commonly used for EDS. The energy resolution (typically $\sim 130 \mathrm{eV}$ at $6 \mathrm{keV}$ ) is often not good enough to resolve emission lines with small energy separations. For example, the difference of the $\mathrm{Si}-\mathrm{K} \alpha$ line $(1.740 \mathrm{keV})$ and $\mathrm{W}-\mathrm{M} \alpha$ line $(1.775 \mathrm{keV})$ is only $35 \mathrm{eV}$. This prevents an accurate determination of the line intensities.

In order to improve the energy resolution, Hollerith et al. [1] have developed SEMEDS mounted on a TES microcalorimeter. We have been developing a STEM-EDS system utilizing a TES X-ray microcalorimeter [2,3]. When we introduced a single pixel TES detector for STEM, we could achieve an energy resolution of $8.9 \mathrm{eV}$ [4]. We obtained the EDS images with this system but the signal-to-noise was not quite good due to the low counting statistic by a single TES detector.

Our new project, the STEM-TES-EDS system analyzes the composition of specimens with high sensitivity and makes it possible to map the distribution of those elements in a specimen. An electron beam scans the specimen and maps the distribution of elements in up to one million pixel images. We need to increase the maximum counting rate to improve the image quality and to complete the measurement within a reasonable time scale. We set a maximum counting rate of $20 \mathrm{kcps}$, an energy resolution less than $10 \mathrm{eV}$ and a wide energy band of $0.5-15 \mathrm{keV}$ to cover most of the atoms with $\mathrm{K}$ to $\mathrm{M}$ fluorescence lines as our requirements.

\section{TES X-ray Microcalorimeter Array for STEM-EDS}

In our STEM-TES-EDS system, the TES detector is set atop an oxygen-free copper rod which is designed by Nagayoshi et al. [5]. The area of the top is $12 \times 12 \mathrm{~mm}^{2}$. When we considered the wiring space and the focal spot size of the polycapillary [6], which was used in this system to increase the solid angle, the maximum area where TES detector can occupy is limited to under $7 \times 7 \mathrm{~mm}^{2}$. We lastly determined the size of the TES detector to be $5.2 \times 5.2 \mathrm{~mm}^{2}$.

The most important requirement of this TES detector is the $20 \mathrm{kcps}$ counting rate. Because the maximum counting rate of our single pixel TES X-ray microcalorimter is about $300 \mathrm{cps}$, we adopted an $8 \times 8$ format, 64 pixel-array. Also we adopted a common biasing scheme [7] to reduce the number of wire harnesses instead of multiplexing. We chose one unit as 8-pixel-TES and read out every one unit by common biasing. In order to operate all pixels at the same time, all pixels are required to have similar superconducting-transition properties. That means the superconducting transition curves coincide with each other and the resistance value of each pixel at the operation point in common bias operation is almost the same. 


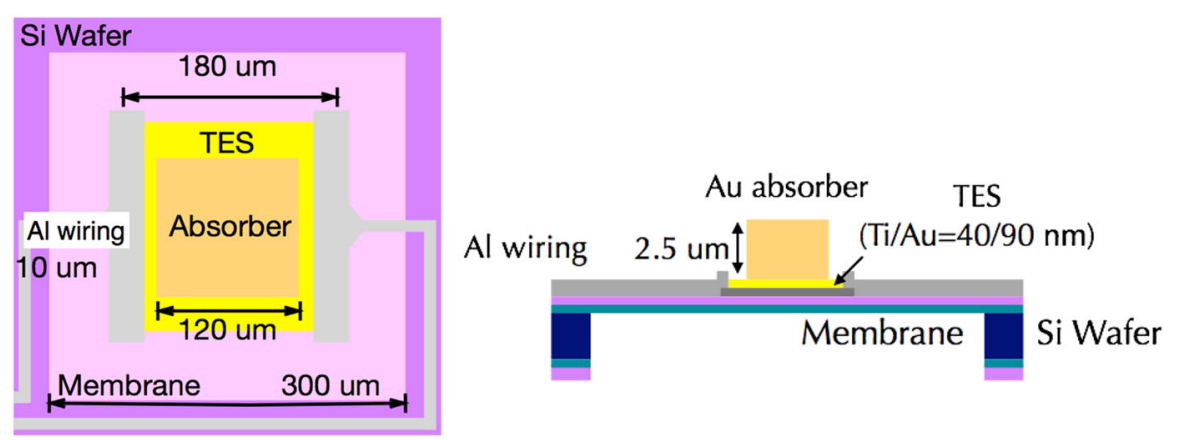

Fig. 1 Top view (left) and cross section view (right) of single pixel TES X-ray microcalorimeter conceptual illustration. The size the of TES, absorber and membrane are $180 \times 180 \mu \mathrm{m}, 120 \times 120 \mu \mathrm{m}, 300 \times 300 \mu \mathrm{m}$, wiring pitch and line are $10 / 10 \mu \mathrm{m}$. The thickness of the absorber and TES (Ti/Au) are $2.5 \mu \mathrm{m}$ and $40 / 90 \mathrm{~nm}$ (Color figure online)

We designed a 64-pixel-TES-array to fulfill the requirements by optimizing the TES parameters, such as transition temperature and heat capacity by the following procedure. The pulse decay time must be shorter than $100 \mu \mathrm{s}$ in order to obtain a maximum counting rate $300 \mathrm{cps} /$ pixel. The pulse decay time [8], energy resolution [9], and saturation energy [9] are given by

$$
\begin{aligned}
\tau_{\mathrm{eff}} & =\frac{C / G}{1+P \alpha / G T} \\
\Delta E & =2.36 \sqrt{\frac{4 k_{\mathrm{B}} T^{2} C}{\alpha} \sqrt{\frac{n}{2}(1+2 \beta)\left(1+M^{2}\right)}}, \beta=5 \times 10^{-5} \alpha^{2.1}, M=0.3 \alpha^{0.5} \\
E_{\mathrm{sat}} & \sim \frac{C T}{\alpha}
\end{aligned}
$$

where $C, G, T, P, k_{\mathrm{B}}, \alpha$, and $\beta$ are, respectively, the heat capacitance, the thermal conductance, the operating temperature, the power, the Boltzmann constant, the sensitivity of the TES, and the current sensitivity. The equations of $\alpha, \beta, G$ are respectively $\alpha=\partial \log R / \partial \log T, \beta=\partial \log R / \partial \log I$ and $G=G_{0} T^{n-1}$. The parameter $G_{0}$ is the thermal conductance at $1 \mathrm{~K}$ and $n$ is the exponent in the power law describing thermal conductance.

We fixed $\alpha, G$ and $n$ based on our previous devices: $\alpha=100, G=0.52 \mathrm{nW} / \mathrm{K}$, $n=3$. We then selected the $T_{\mathrm{c}}$ and $C$ from Eqs. (1)-(3) as $200 \mathrm{mK}$ and $0.6 \mathrm{pJ} / \mathrm{K}$ to meet the requirements.

We then designed the size and the thickness of TES X-ray microcalorimeter. Figure 1 shows a conceptual image of the TES X-ray microcalorimeter and its size. We fabricated the TES X-ray microcalorimeter array using an in-house fabrication process [10]. We use 4 inch $\mathrm{Si}$ wafer with deposited $\mathrm{SiO}_{2}$ and $\mathrm{SiN}_{x}$. The first step is to remove $\mathrm{SiN}_{x}$ and $\mathrm{SiO}_{2}$ on the back of the $\mathrm{Si}$ substrate, for the later Si bulk etching. The TES is created by sputtering and wet etching of Ti and Au. We add Al electrodes in the same 

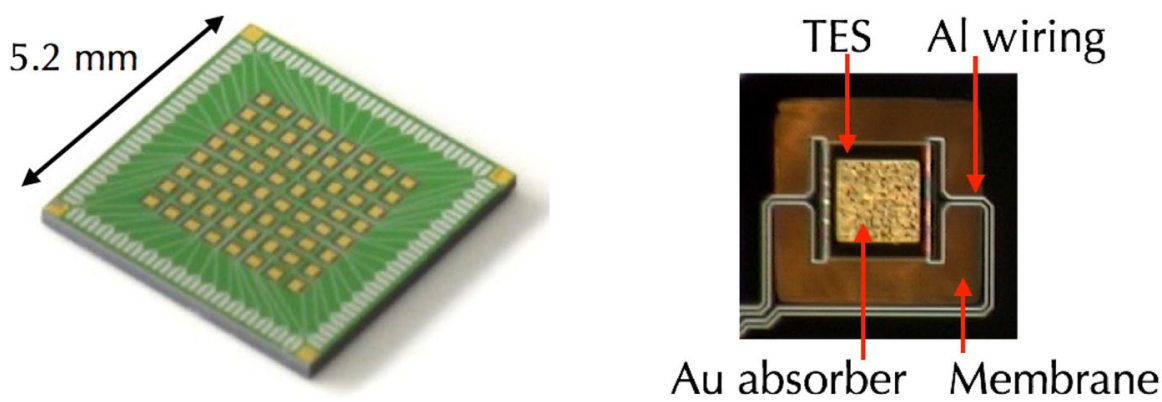

Fig. 2 64-pixel-TES-array that we fabricated (left), Microscope photograph of the single pixel TES X-ray microcalorimeter from top (right) (Color figure online)

way and followed by Au absorber by EB vapor deposition. Lastly the bulk Si of the membrane area will be removed by DRIE etching. We show the 64-pixel-TES-array that we fabricated and a microscope photograph of a single pixel TES X-ray (Fig. 2).

\section{Performance of This TES X-ray Microcalorimeter Array}

There are many things to evaluate the performance of this TES X-ray microcalorimeter array. For example, the properties of $R(T)$ and IV-curves, transition temperature, pulse decay time, energy resolution, saturation energy, and the performance of TES with high counting rate. We did the $R T$ and IV measurement and X-ray irradiation test to confirm the performances of this device.

\subsection{Experiment Setup}

We used a cryogen-free dilution refrigerator at JAXA with a lowest achievable temperature of $\sim 60 \mathrm{mK}$. The 64-pixel-TES-array shown in Fig. 2 and SQUID amplifiers are placed on a cold stage with $\mathrm{RuO}_{2}$ thermometer which is readout and controlled by an Lakeshore $\mathrm{AC}$ resistance bridge 370 and $100 \mathrm{~m} \Omega$ resistance heater. $\mathrm{An}{ }^{55} \mathrm{Fe}$ isotope is also mounted at a distance of $\sim 3 \mathrm{~mm}$ from the TES to serve as an X-ray source.

\subsection{The Superconducting-Transition Properties}

We chose the 7 pixels of one unit and measured $R(T)$ and $\mathrm{I}-\mathrm{V}$ curves of the 7 pixels to confirm the superconducting-transition properties (Fig. 3). The superconducting transition of 7 pixels is almost uniform at about $200 \mathrm{mK}$ as designed. We also see small differences among pixels. The dispersion is as small as $0.6 \mathrm{mK}$. This is smaller than the transition range $(\sim 5 \mathrm{mK})$. The dispersion of the TES-array resistances at the operation point in a common bias operation can be estimated from the I-V curves. It is estimated to be less than $10 \%$. These differences are small enough to operate the TES X-ray microcalorimeter with common bias scheme. 

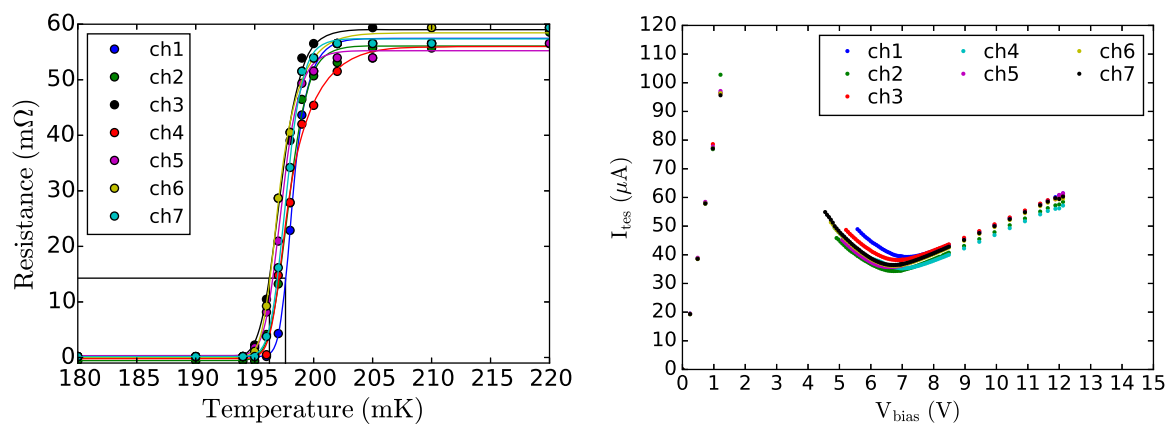

Fig. $3 R(T)$ of 7 pixels TESs in one unit (left), $V_{\text {tesb }}$ and $I_{\text {tes }}$ curve of 7 pixels TESs in one unit (right) (Color figure online)
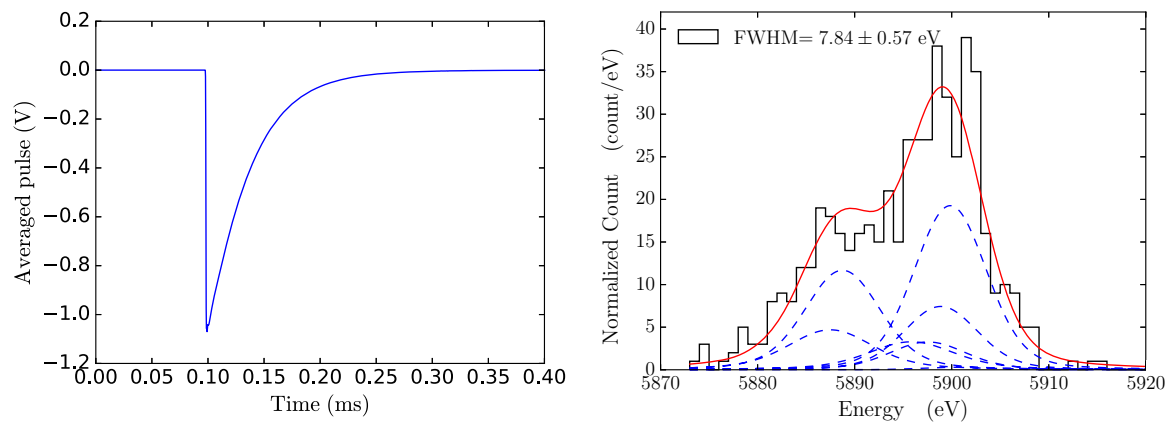

Fig. 4 Averaged pulse which obtained by one pixel (left), Mn K $\alpha$ spectrum of ${ }^{55} \mathrm{Fe}$ which obtained by one pixel (right) (Color figure online)

\subsection{X-ray Irradiation Test}

We measured the X-ray energy resolution and decay time of one pixel to confirm the performance. The thermal bath temperature is set at $150 \mathrm{mK}$. A TES bias current is set at $715 \mu \mathrm{A}$ which means that it corresponds to $54 \%$ of transition curve $(40 \mathrm{~m} \Omega)$. The two key experimental results are shown Fig. 4. First, the pulse decay time was $40 \mu \mathrm{s}$. With this decay time, we expected to achieve $50 \mathrm{kcps}$ by the 64 pixels array. Second, the energy resolution was $7.8 \mathrm{eV}$ at $5.9 \mathrm{keV}$ in full width at half maximum (FWHM) as shown in the right side of Fig. 4. Mn $\mathrm{K} \alpha$ and $\mathrm{K} \beta$ are easily separated. When we zoom in on the $\mathrm{K}$ alpha-line, we see the structure of $\alpha 1$ and $\alpha 2$. We could measure the values of $\alpha, C$ and $G$ at operation point and they are, respectively $34,0.56 \mathrm{pJ} / \mathrm{K}$ and $0.22 \mathrm{nW} / \mathrm{K}$. The value of $C$ was almost same as designed and the values of $\alpha$ and $G$ were smaller than the designed values but we could achieve the requirements.

\section{Conclusion}

We designed a 64-pixel TES array to fulfill the requirements for a STEM-EDS and fabricated it using an in-house process. We evaluated some of the performances of the 
TES detector. We found the TES array can be operated with common bias scheme, with a single pixel decay time of $\sim 40 \mu$ s while the requirement is $<100 \mu$ s to obtain $300 \mathrm{cps} /$ pixel. The energy resolution is about $7.8 \mathrm{eV}$ at $5.9 \mathrm{keV}$ while the requirement is less than $10 \mathrm{eV}$. There are two remaining issues where the performance must be confirmed by the following procedure. We will verify the energy range by irradiating multi-color X-rays onto the TES X-ray microcalorimeter array and evaluate the performance of the TES X-ray microcalorimeter array with high counting rate by irradiation tests using an X-ray generator. We will then mount the TES X-ray microcalorimeter array on a STEM and operate the STEM-EDS system.

Acknowledgments This work was financially supported by SENTAN, Japan Science and Technology Agency (JST).

Open Access This article is distributed under the terms of the Creative Commons Attribution 4.0 International License (http://creativecommons.org/licenses/by/4.0/), which permits unrestricted use, distribution, and reproduction in any medium, provided you give appropriate credit to the original author(s) and the source, provide a link to the Creative Commons license, and indicate if changes were made.

\section{References}

1. C. Hollerith, D. Wernicke, M. Bühler, Fv Feilitzsch, M. Huber, J. Höhne, T. Hertrich, J. Jochum, K. Phelan, M. Stark, B. Simmnacher, W. Weiland, W. Westphal, Nucl. Instrum. Methods Phys. A 520, 606-609 (2004). doi:10.1016/j.nima.2003.11.327

2. T. Hara, K. Tanaka, K. Maehata, K. Mitsuda, N.Y. Yamasaki, M. Ohsaki, K. Watanabe, X. Yu, T. Ito, Y. Yamanaka, J. Electron Microsc. 59(1), 17-26 (2010)

3. K. Tanaka, A. Odawara, A. Nagata, Y. Baba, S. Nakayama, S. Aida, T. Morooka, Y. Homma, I. Nakai, K. Chinone, Nonmembers, IEICE Trans. Electron E92-C(3), 334-340 (2009)

4. K. Maehata, T. Hara, K. Mitsuda, M. Hidaka, K. Tanaka, Y. Yamanaka, J. Low Temp. Phys. (2015). doi:10.1007/s10909-015-1361-3

5. K. Nagayoshi, T. Hayashi, H. Muramatsu, N.Y. Yamasaki, K. Mitsuda, T. Hara, J. Low Temp. Phys., in this Special Issue

6. A. Takano, K. Maehata, N. Iyomoto, K. Yasuda, H. Maeno, K. Shiiyama, K. Tanaka, J. Low Temp. Phys. (2015). doi:10.1007/s10909-015-1350-6

7. R. Yamamoto, K. Sakai, K. Nagayoshi, T. Hayashi, H. Muramatsu, Y. Nakashima, K. Mitsuda, N.Y. Yamasaki, Y. Takei, M. Hidaka, S. Nagasawa, K. Maehata, T. Hara, J. Low Temp. Phys., in this Special Issue

8. K.D. Irwin, Appl. Phys. Lett. 66, 1998 (1995)

9. N. Kenichiro, S. Kazuhiro, M. Kazuhisa, Y.Y. Noriko, T. Yoh, M. Keisuke, I. Naoko, E. Shohei, T. Akira, M. Makoto, H. Toru, IEICE Trans. Electron. E98-C(3), 186-191 (2015)

10. H. Muramatsu, Master's thesis, University of Tokyo, 2016 